\title{
Charge deep-level transient spectroscopy study of high-energy-electron- beam-irradiated hydrogenated amorphous silicon
}

\author{
A. Klaver \\ DIMES-ECTM, Delft University of Technology, P.O. Box 5053, 2600 GB Delft, The Netherlands \\ V. Nádaždy \\ Institute of Physics of SAS, Dúbravská cesta 9, 84511 Bratislava, Slovak Republic \\ M. Zeman and R. A. C. M. M. van Swaaij ${ }^{\text {a) }}$ \\ DIMES-ECTM, Delft University of Technology, P.O. Box 5053, 2600 GB Delft, The Netherlands
}

(Received 7 February 2006; accepted 10 June 2006; published online 14 July 2006)

\begin{abstract}
We present a study of changes in the defect density of states in hydrogenated amorphous silicon $(a-\mathrm{Si}: \mathrm{H})$ due to high-energy electron irradiation using charged deep-level transient spectroscopy. It was found that defect states near the conduction band were removed, while in other band gap regions the defect-state density increased. A similar trend is observed for $a-\mathrm{Si}: \mathrm{H}$ which has been subjected to light soaking, but in that case the majority of defect states are created around midgap, whereas with electron-beam degradation more defect states are created near the valence-band tail. (C) 2006 American Institute of Physics. [DOI: 10.1063/1.2221876]
\end{abstract}

Hydrogenated amorphous silicon $(a-\mathrm{Si}: \mathrm{H})$ solar cells show great promise for space application, particularly in high-radiation missions, due to their relatively high radiation tolerance and low-temperature annealing properties. ${ }^{1,2}$ Various studies have addressed the degradation behavior of $a$ -Si:H due to high-energy charged particle irradiation, and it is generally accepted that changes in the defect density of states govern the degradation, although trapping of the incident charge on preexisting defects is also considered. ${ }^{3-5}$ The underlying degradation mechanism, however, is still under debate. Danesh et al., ${ }^{6}$ amongst others, showed that there is a strong similarity between high-energy charged particle irradiation and the Staebler-Wronski effect, ${ }^{7}$ i.e., reversible changes in electronic properties of $a-\mathrm{Si}: \mathrm{H}$ under light exposure. In that case, it is expected that the defect-state distribution after light soaking is similar to the distribution after high-energy electron-beam irradiation. In this letter we report on changes in defect-state distribution of $a-\mathrm{Si}: \mathrm{H}$ due to electron-beam irradiation measured by charge deep-level transient spectroscopy ( $Q$-DLTS). These results are compared to findings from light-soaking experiments in order to investigate the reported similarities in the defect-creation mechanism further.

$Q$-DLTS has been utilized to study the defect-state distribution in as-deposited as well as in light-soaked $a-\mathrm{Si}: \mathrm{H}$. This technique gives direct information about the energy distribution of the gap states and is very sensitive to changes in the gap-state distribution. According to the defect-pool model by Powell and Deane, ${ }^{8}$ at thermal equilibrium three distributions of amphoteric defect states are identified: positively charged, $D_{h}$, neutral, $D_{z}$, and negatively charged, $D_{e}$, defect states. Recently, a microscopic model ${ }^{9}$ was proposed, in which the $D_{z}$ states are linked to dangling bonds, the $D_{h}$ states are associated with complexes formed by ionized hydrogen molecules weakly bonded to dangling bonds, and the $D_{e}$ states are ascribed to floating bonds. In the same article a

\footnotetext{
a) Author to whom correspondence should be addressed; electronic mail:
} r.vanswaaij@dimes.tudelft.nl four stage process was proposed for the light-induced degradation of $a$-Si:H: (i) reduction of $D_{h}$-state density, followed by (ii) an increase of the $D_{z}$ and $D_{e}$-state densities and continuation of the $D_{h}$-state density reduction, subsequent (iii) dominant increase in $D_{z}$-state density, while other defectstate densities do not alter significantly, and finally (iv) the increase in $D_{z}$ and $D_{e}$-state densities. In a typical $Q$-DLTS spectrum of intrinsic $a-\mathrm{Si}: \mathrm{H}$ using a rate window of $100 \mathrm{~s}^{-1}$, the signal at about 320,390 , and $430 \mathrm{~K}$ is related to $D_{h}, D_{z}$, and $D_{e}$ states, respectively. ${ }^{10}$

$Q$-DLTS measurements were performed on $a$-Si:H based metal-oxide-semiconductor (MOS) structures, consisting of a $1-\mu \mathrm{m}$-thick $a$-Si:H layer deposited on $n^{++}$crystalline Si. The fabrication method of the MOS structure is described by Durný et al. ${ }^{11}$ The measurements were carried out using a bias voltage of $-3 \mathrm{~V}$, excitation pulses of $6 \mathrm{~V}$, and a rate window of $100 \mathrm{~s}^{-1}$. Prior to electron-beam irradiation or light soaking, the samples were annealed at $200{ }^{\circ} \mathrm{C}$ for 30 min. A Van de Graaff accelerator, with a beam current density of $1.5 \times 10^{12}$ electrons $\mathrm{cm}^{-2} \mathrm{~s}^{-1}$, was employed for the $1-\mathrm{MeV}$ electron irradiation using a fluence of $2 \times 10^{15}$ and $2 \times 10^{16}$ electrons $\mathrm{cm}^{-2}$. Light soaking was performed for 6 and 60 min using a red light laser having a wavelength of $670 \mathrm{~nm}$ and a power density of $1 \mathrm{~W} \mathrm{~cm}^{-2}$. These conditions lead to comparable changes in the amplitude of the $Q$-DLTS spectrum as for electron-beam-irradiated samples.

Figure 1 shows the $Q$-DLTS spectra of three investigated samples in their annealed state, i.e., before either electronbeam irradiation or light soaking. For the three samples similar $Q$-DLTS spectra are found, which show the typical shape for the annealed state, indicating that all samples have similar properties for the oxide and $a$-Si:H layers. Figure 2 shows the $Q$-DLTS spectra for two electron-irradiation experiments as well as the annealed state. Following irradiation, a decrease in signal for temperatures below $350 \mathrm{~K}$ and an increase for temperatures above $350 \mathrm{~K}$ are found, concluding that the irradiation leads to a decrease in the $D_{h}$-state density and an increase of the $D_{z^{-}}$and $D_{e^{- \text {state }}}$ densities. Increasing the fluence leads to a further increase of the 


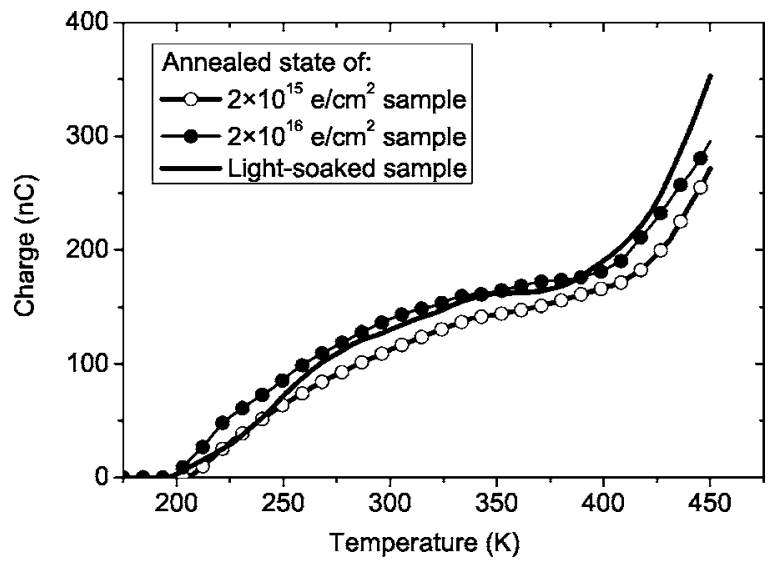

FIG. 1. $Q$-DLTS spectra of the samples used for light soaking and electronbeam irradiation in the annealed state.

$D_{z}$-state density and an even larger increase of the $D_{e}$-state density while lowering the $D_{h}$-state density further.

When analyzing the data, we need to take into account that the oxide and the $n^{++}$-doped crystalline silicon substrate are possibly affected by the irradiation also. We consider three effects that irradiation-induced defects or trapped charge in the oxide can have on the $Q$-DLTS signal: (i) an increase in the leakage current through the oxide, (ii) a change in the voltage drop over the oxide, and linked to this effect, (iii) a change in the band bending in the $a-\mathrm{Si}: \mathrm{H}$. The leakage current can be identified in the measurements by the fact that it scales exponentially with temperature. However, no significant increase in leakage current is observed in the measurements. The influence of the latter two effects on the $Q$-DLTS signal is small, because an increase in defect density of the oxide would invariably lead to an increase in trap-assisted leakage current, which is the dominant leakage current mechanism for this type of oxide. ${ }^{12}$ The $n^{++}$-doped wafer acts as a hole barrier. Increasing the defect density in the crystalline silicon would lead to a small increase in the hole current, but it is expected that the hole current will stay much lower than the total current which contributes to the transient charge. Finally, the irradiation-induced changes observed in the $Q$-DLTS spectra are reversible by a 30 -min anneal step at $200{ }^{\circ} \mathrm{C}$, indicating that there is no significant permanent degradation of the sample and that the degrada-

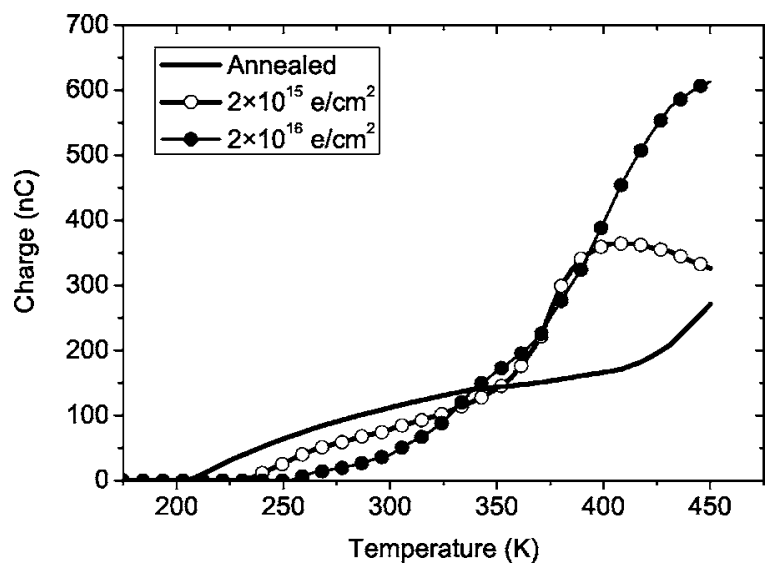

FIG. 2. $Q$-DLTS spectra of the samples subjected to electron-beam irradiation, including the annealed state.
FIG. 3. Difference in $Q$-DLTS signal following light soaking and electronbeam degradation as compared to their annealed state.

tion observed is due to reversible changes in the $a-\mathrm{Si}: \mathrm{H}$ material.

Figure 3 compares the difference between the degraded and annealed $Q$-DLTS spectra following electron-beam irradiation and light soaking. A positive difference indicates an increase in defect-state density. Both degradation mechanisms show a similar general trend in the changes in defectstate densities: an increase in $D_{z^{-}}$and $D_{e}$-state densities along a decrease in $D_{h}$-state density. However, the lightsoaked sample shows a larger decrease in the $D_{h}$-state density and a stronger increase in the $D_{z}$-state density (full line in Fig. 3). On the other hand, in the electron-beam-irradiated samples, the $D_{e}$-state density increases more (full symbols in Fig. 3). In order to quantify the changes, we have used computer simulations following the procedure as described by Nádaždy et al. ${ }^{13}$ where the defect density of states is extracted from the $Q$-DLTS spectra. The results are given in Table I.

The last effect can be understood by considering the difference in the electron-hole pair generation rates between both degradation methods. Biswas and Pan ${ }^{14}$ concluded from theoretical calculations, in which the degradation mechanism is linked to recombination processes in the material, that the ratio between the increases in $D_{z^{-}}$and $D_{e}$-state densities during light soaking is dependent on the light intensity, i.e., the electron-hole (e-h) pair generation rate and corresponding recombination rate. They concluded that at higher light intensities, increases in $D_{e}$-state density are more pronounced. With electron-beam degradation, one incident electron generates a funnel of e-h pairs along its path through the material. In this funnel the generation rate is much higher, leading to a larger local recombination rate. During light soaking, each photon generates one e-h pair only and the pairs are generated approximately uniformly (in time and space) in-

TABLE I. The extracted defect-state density values of the $Q$-DLTS samples following electron-beam irradiation (EI) with the highest fluence and light soaking (LS) for the longest time and their corresponding as-deposited defect-state densities, AS(EI) and AS(LS), respectively.

\begin{tabular}{lccc}
\hline \hline \multicolumn{1}{c}{ Sample } & $D_{h}\left(\mathrm{~cm}^{-3}\right)$ & $D_{z}\left(\mathrm{~cm}^{-3}\right)$ & $D_{e}\left(\mathrm{~cm}^{-3}\right)$ \\
\hline AS(LS) & $2.1 \times 10^{16}$ & $1.1 \times 10^{16}$ & $1.9 \times 10^{16}$ \\
LS & $>1 \times 10^{15}$ & $4.5 \times 10^{16}$ & $2.3 \times 10^{16}$ \\
AS(EI) & $1.9 \times 10^{16}$ & $8.9 \times 10^{15}$ & $1.8 \times 10^{16}$ \\
EI & $2.2 \times 10^{15}$ & $2.1 \times 10^{16}$ & $6.4 \times 10^{16}$ \\
\hline \hline
\end{tabular}


side the material. In addition, when using the microscopic model proposed by Nádaždy and Zeman, ${ }^{9}$ we can conclude that due to the slower annihilation rate of $D_{h}$ states in electron-beam-irradiated $a-\mathrm{Si}: \mathrm{H}$, less mobile hydrogen is released, which in turn suppresses the hydrogen-floating-bond interactions, leaving a higher $D_{e}$-state density. The differences in generation rates can also explain the difference in the annihilation rate of $D_{h}$ states between both degradation methods. The foremost $D_{z^{-}}$and $D_{e}$-state creation process is e-h pair recombination leading to breaking of weak bonds and this process has a square dependence on the e-h pair generation rate. ${ }^{14}$ If the annihilation rate of the $D_{h}$ states varies slower than the square of the generation rate (for instance, $D_{h}$ states might be annihilated by electron capture at the corresponding defect), an increase in generation rate will result in higher creation rate of $D_{z}$ and $D_{e}$ states as compared to the annihilation rate of the $D_{h}$ states, as is observed in Fig. 3.

In summary, we have investigated the $1-\mathrm{MeV}$ electronbeam degradation of $a$-Si:H using $Q$-DLTS. A decrease in $D_{h^{-}}$-state density is observed, while the $D_{z^{-}}$and $D_{e^{-}}$-state densities increase, similar to light-induced changes in the defectstate density. Increasing the fluence leads to a more pronounced effect. $Q$-DLTS measurements reveal some differences not reported so far: In electron-beam-irradiated samples there tends to be a preference for an increase in the $D_{e}$-state density instead of the $D_{z}$-state density. This difference can be explained by considering the differences in the e-h pair generation rate between both degradation methods.

The authors acknowledge the support of STW, SenterNovem, and Dutch Space. This work was partially supported by Slovakian grant agencies APVV under Contract No. 51013904 and VEGA under Contract No. 2/4100/04. The authors thank Martijn Tijssen and Marinus Hom for their technical assistance.

${ }^{1}$ G. Grigorieva, M. Kagan, V. Nadorov, and K. Zvyagina, in Proceedings of the Third World Conference on Photovoltaic Energy Conversion, Osaka, Japan, 11-18 May 2003, edited by K. Kurokawa, L. L. Kazmerski, B. McNelis, M. Yamaguchi, C. Wronski, and W. C. Sinke (IEEE, New York, 2003), p. 734.

${ }^{2}$ J. Kuendig, M. Goetz, X. Niquille, A. Shah, S. Vaccaro, J. Mosig, L. Gerlach, P. de Maagt, and E. Fernandez, Proceedings of the 28th IEEE Photovoltaic Specialists Conference, Anchorage, AK, 15-22 September 2000 (IEEE, Piscataway, NJ, 2001), p. 1079.

${ }^{3} \mathrm{Q}$. Wang, K. Lord, and J. R. Woodyard, Proceedings of the 28th IEEE Photovoltaic Specialists Conference, Anchorage, AK, 15-22 September 2000 (IEEE, Piscataway, NJ, 2001), p. 1057.

${ }^{4}$ J. R. Srour, G. J. Vendura, D. H. Lo, C. M. C. Toporow, M. Dooley, R. P. Nakano, and E. E. King, IEEE Trans. Nucl. Sci. 45, 2624 (1998).

${ }^{5}$ J. E. Granata, T. D. Sahlstrom, P. Hausgen, S. R. Messenger, R. J. Walters, and J. R. Lorentzen, Proceedings of the 31st IEEE Photovoltaic Specialist Conference, Orlando, FL, 3-7 January 2005 (IEEE, Piscataway, NJ, 2005), p. 607.

${ }^{6}$ P. Danesh, B. Pantchev, I. Savatinova, E. Liarokapis, S. Kaschieva, and A. G. Belov, Vacuum 69, 79 (2003).

${ }^{7}$ D. L. Staebler and C. R. Wronski, Appl. Phys. Lett. 31, 292 (1977).

${ }^{8}$ M. J. Powell and S. C. Deane, Phys. Rev. B 48, 10815 (1993).

${ }^{9}$ V. Nádaždy and M. Zeman, Phys. Rev. B 69, 165213 (2004).

${ }^{10}$ V. Nádaždy, R. Durný, and E. Pinčik, Phys. Rev. Lett. 78, 1102 (1997).

${ }^{11}$ R. Durný, E. Pinčik, V. Nádaždy, M. Jergel, J. Shimizu, M. Kumeda, and T. Shimizu, Appl. Phys. Lett. 77, 1783 (2000).

${ }^{12}$ A. Candelori, M. Ceschia, A. Paccagnella, J. Wyss, D. Bisello, and G. Ghidini, IEEE Trans. Nucl. Sci. 48, 1735 (2001).

${ }^{13}$ V. Nádaždy, R. Durný, and M. Zeman, J. Non-Cryst. Solids 352, 1059 (2006).

${ }^{14}$ R. Biswas and B. C. Pan, J. Non-Cryst. Solids 299-302, 507 (2002). 